\title{
Coal Mine Safety Inspection System Based on Real-Time Online Intelligent Control System Intemor
}

\author{
Yuanzhi Huang \\ Lanzhou Resource and Environment Voc-Tech College, Electrical and Mechanical Department, Lanzhou, \\ 730021, China.
}

Keywords: Real-Time, Online Intelligent Control System, Intemor Technology, Coal Mine Safety Inspection System

\begin{abstract}
Coal mine resources are important natural mineral resources in our country, in fact, one of the essential resources in economic development, industrial production, electric energy production and people's daily life. In the process of coal mine production and operation, the application of intelligent and automatic control technology can effectively improve the safety and production efficiency of coal mine production and operation. The real-time on-line intelligent control system INTEMOR system is an advanced intelligent management control system in coal mine safety inspection system.
\end{abstract}

\section{Introduction}

At present, with the improvement of our society and economy level and the development of science and technology, the safety problems of coal mining and other natural mineral production operations have been improved very well, especially the application of intelligent operating system and intelligent control system has greatly improved the safety and accuracy of coal mining operations. The research of mining technology and the application of modern science and technology have greatly enhanced the safety and function of coal seam, geological structure, roadway excavation, support and so on. Among them, the safety of coal mine operation is a part of people's attention from beginning to end. It is well known that coal mine production and mining operations are extremely dangerous, involving many underground geological structure threats, aquifer leakage threats, dust explosion threats, underground collapse threats and so on. Therefore, coal mining operations must pay attention to the real-time detection of safety risks to ensure the safety of coal mining operations.

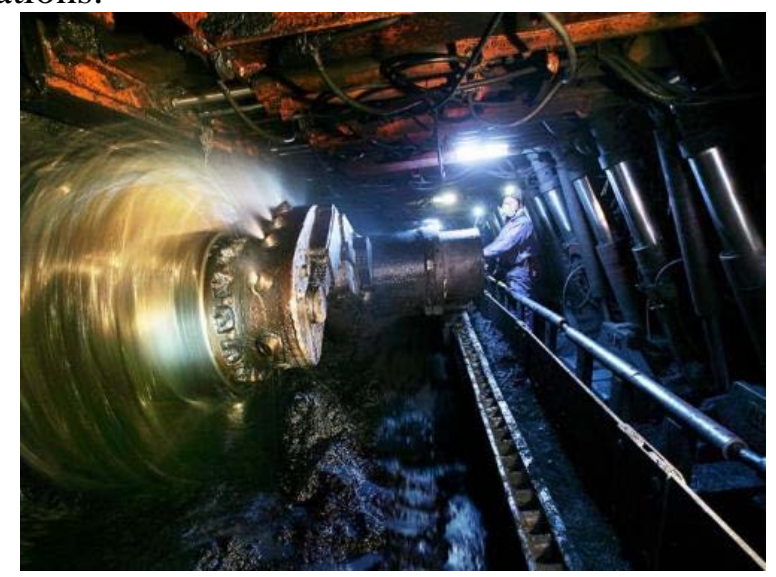

Figure 1 Coal mining

\section{Definition of INTEMOR System for Real-time Online Intelligent Control System}

The real-time online intelligent control system INTEMOR, also known as the real-time online intelligent control Intemor system, is an online intelligent control system developed by the SinoCanada Joint Center for Intelligent Control Engineering. In fact, it will include intelligent system of 
expert system, intelligent detector, automatic translator and information display equipment, network technology, intelligent detector, communication technology, automatic control technology, timely data analysis and processing technology and database technology.

\section{Content of INTEMOR System of Real-time Online Intelligent Control System}

At the technical level, it is a system that integrates the distributed control system, the management system and the knowledge and information data integration system. The main application scope includes the intelligent real-time monitoring and safety warning system in industrial production, chemical production and mining of mineral resources. The first is to carry on the real-time running state of the running equipment, the acquisition and arrangement of the data, the data engineering, the data conversion and the analysis and compression of the information data in the production process. Data engineering and data conversion mainly refers to the acquisition and collection of effective and swimming information data in raw data information, mainly including data filtering, data standardization, taking mean and other related information extraction, and data compression is a storage method of information data, which can provide accurate data support for subsequent production and the control of the whole project. The second is to be able to achieve data acquisition and construction of knowledge database, but also to use knowledge base search tools for professional technology, methods, suggestions and so on, which belongs to the comprehensive data compression error number. A large number of expert knowledge and technology are brought together to form an integrated system with a high level of expertise and a large amount of knowledge data. Its database contains a large number of specialized technology and related knowledge, which can effectively carry out the establishment of the knowledge base of coal mining, production supervision, safety risk prediction, diagnosis, safety accident early warning, online operation guidance and the use of knowledge structure to coordinate the knowledge structure of various parts of intelligent system and information data. Thirdly, the Intmore system integrates most of the event and safety risk factors, which are based on the event inference machine, the forward thrust machine and the response inference machine. The forward inference machine is mainly used to monitor and check the mining function of the coal mine. If the warning signal is generated, it is necessary to predict the staff and the safety accident. Fourth, the intelligent production accident supervision system, in fact, contains the field image acquisition, real-time on-line monitoring and automatic switching and other functions of the process, mostly using information technology and display related accident information data input, and form a fixed database to facilitate the analysis and careful study of the accident, is a "black box" in coal mine production, can provide some data support for experts to handle safety accidents.

\section{Application of Intemor System in Coal Mine Safety Detection System}

In the process of coal mining, because of its harsh working environment and its relatively closed space and the influence of various materials, dust, metal and other elements, its dangerous technology is extremely high, and the application of real-time on-line intelligent control and control system in coal mine safety production can collect and store the accident information data, and through the continuous accumulation and analysis of storage gradually form the database of the system, combined with the improvement of actual real-time monitoring mining operation conditions and actual mining mode, the modern coal mining is realized, and the safety and intelligent level of mining operation can be improved to the greatest extent.

\section{Application Analysis of Coal Mine Safety Inspection System in Real-Time On-Line Intelligent Control System Intemor}

\subsection{Collection and Processing of Data Information By Intemor System in Coal Mine Safety Inspection System}

In order to realize the application of the real-time on-line intelligent control system, the accident 
forecast of INTEMOR system is based on the analysis and processing of the collected data, so it is necessary to collect and collate the data, for example, in the coal mining environment about the concentration of carbon monoxide and methane in the air. In fact, the application of the real-time intelligent control and control system in the safety detection system of safety production is mainly to obtain and accumulate the accident private ownership through the platform, and gradually monitor and warn the safety accident accurately, and apply the safety production management system to the advanced technical means and technical equipment for emergency treatment, so as to eliminate the hidden danger of the safety accident, which is mainly divided into the collection and analysis of the data information, the collection and processing of private knowledge, the common knowledge, and the formation and establishment of the database through the display window editor, so as to provide the security and stability guarantee for the coal mine production.

The real-time intelligent control and control of INTE MOR system for the collection of information data is mainly through two ways of data collection and collation.First, through the use of manpower for the input of data information, the realization of manual testing, beam tube detection, manual inspection of all the acquired information into the database, the second is based on the advanced monitoring equipment and detection instruments to talk about data collection, and combined with the field industrial database can extract the data to achieve the purpose of information collection. Most of the information data are obtained through sensors and monitoring devices, but the collection and processing of the data information are stored in the database, and then through the call to achieve data analysis, and finally obtain effective data information for coal mine operation environment related safety risks and risk information.

In the developer's data acquisition program, the real-time on-line system of the system will transmit the real-time data to INTEMODRIVR and INTEMODRIVR after obtaining the data information.

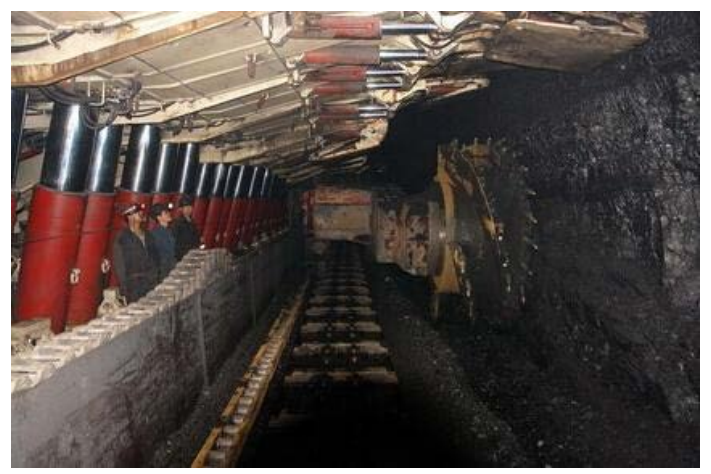

Figure 2 Underground coal mines

In the framework of this system structure, it can be clearly seen that the functions of Intmore data acquisition and preprocessing mainly carry out the collection of on-site data information, and make it form a large amount of data through the service to realize the distribution of information, one is to be able to transmit the underground data information to the Intmore system service period and to display the real-time data, the other is to record the data in the database after the analysis, so as to facilitate the use of the Intmore remote data monitoring module of the real-time online intelligent control system.

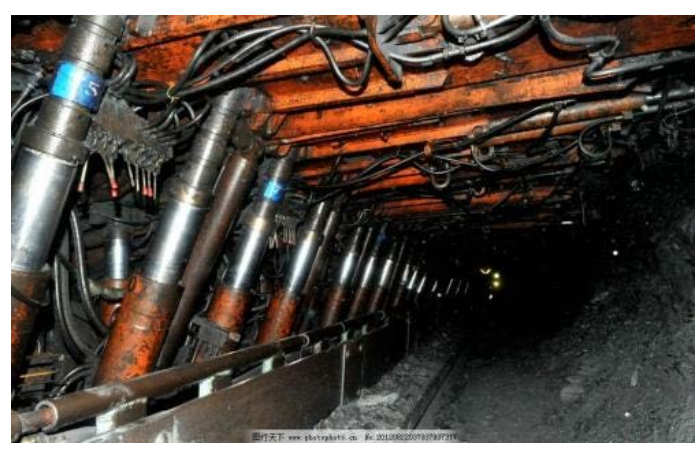

Figure 3 Coal mining 


\subsection{Real-Time Online Intelligent Control of Intemor Systems for Private and Public Knowledge Acquisition}

The private and public knowledge collected by the Intmore system software can be obtained through many channels, and its main function is to compare and analyze the downhole information data obtained by the real-time online intelligent control system, and use it as the basis for the activation of the early warning and emergency measures of the coal mine safety system.

\section{Conclusion}

Real-time on-line intelligent control and control INTEMOR system is an advanced system for real-time on-line monitoring and early warning of safety accidents. It can carry out safety risk detection and management control of emergency measures in coal mines, including gas system, system basis, supervision and inspection, etc. Its main advantage lies in the use of expert system development tools to transform knowledge into a framework, through the side of the knowledge structure into information technology software program, and then achieve multi-functional application and accuracy improvement.

\section{Acknowledgements}

Research project of Gansu Higher Education Institute, project number: 2018C-25

\section{References}

[1] Bai, Jingzhi., Wang, Yongbao., Xu, Guohua. Application of intelligent safety monitoring system in Wanglou coal mine. Shandong Coal Technology, vol. 219, no. 11, pp. 94-96, 2018.

[2] Zhang, Yi. Research on intelligent control system of coal mine air compressor based on PLC. Coal Mining Machinery, vol. 39, no. 364, pp. 163-165, 2018.

[3] Yang, Yang., Qin, Xiaohui., Fu, Jianglong. Design of intelligent monitoring and early warning system for coal mine safety. IoT Technologies, vol. 8, no. 12, pp. 23-24+27, 2018.

[4] Choi, Po-wen. application of intelligent safety management system in coal mines. Inner Mongolia Coal Economy, vol. 209, no. 09, pp. 5+19, 2016. 